\title{
Bioluminescence Resonance Energy Transfer (BRET) Based Nanostructured Biosensor for Tear Glucose Detection
}

\author{
Denghuang Zhan', Longyi Chen', Wai Hei Tse², Longyan Chen', Jin Zhang1, 2 \\ ${ }^{1}$ Department of Chemical \& Biochemical Engineering, University of Western Ontario \\ London, Ontario, Canada N6A 5B9 \\ ${ }^{2}$ Department of Medical Biophysics, University of Western Ontario \\ London, Ontario, Canada N6A 5B9 \\ jzhang@eng.uwo.ca
}

\begin{abstract}
The monitoring and management of blood glucose levels are key elements for people with diabetes to maintain their health. Here, we describe a bioluminescence resonance energy transfer (BRET) sensor for non-invasively detecting glucose molecules. The sensor is assembled by conjugating quantum dots $\mathrm{CdTe}(\mathrm{PL} \approx 565 \mathrm{~nm})$, which is used as the acceptor, with a recombinant protein containing the bacterial glucose binding protein (GBP), at the N-terminal and a bioluminescent protein Renilla luciferase (Rluc), used as the donor, which is at the C-terminal. The distance between the BRET pair is initially far. In the presence of glucose, GBP binds glucose in a highly specific manner and the conformational change of resultant GBP brings a closer distance between the Rluc and QDs, results an increasing of the emission intensity of the QDs. The bioluminescence intensity of both around 470nm and 565nm are observed. The ratio of the acceptor (QDs) and the donor (Rluc) are also observed to increase with the increasing of the glucose concentration. This study laid a technical foundation for further exploration of non-invasive measurement systems for tear glucose.
\end{abstract}

Keywords: Bioluminescence resonance energy transfer (BRET), Luciferase, Glucose binding protein, Quantum dots, Tear glucose, Non-invasive biosensor

\section{Introduction}

As the numbers of diabetes patients increased so fast worldwide, the desire of constructing glucose sensor especially the non-invasive types have drawn numerous attentions[1]. Body fluids such as urine [2-4], sweat [5-8] and tear [9-12] thus have been widely used as testing samples. It has been found that tear glucose level is around 5 times higher in diabetes patients than in normal people and the tear glucose has been identified to have a positive correlation with the blood glucose based on previous research[13-15]. In order to test the extremely low concentration of glucose from the tiny amount of the tear sample, bioluminescence resonance energy transfer (BRET), which is a form of fluorescence resonance energy transfer (FRET) has been used in bioimaging and biosensing systems because of its high sensitivity for distance change[16-19]. Glucose/galactose-binding protein (GGBP) is a bacterial periplasmic-binding protein that exhibits a hinge motion and results of conformational change upon binding glucose or galactose molecules[20, 21]. Though a lot of research has been done using Glucose/galactose binding protein and its mutation to build biosensor for detecting glucose molecules [22-25], there is no report on the combination of GBP with BRET to our best knowledge.

Here, we report a novel glucose sensing system strategy which is based on the fluorescence changes of the probe as the protein undergoes a structural change on binding. The recombinant fusion protein consists of bacterial glucose binding protein (GBP), at the N-terminal and a bioluminescent protein Renilla luciferase (Rluc) at the C-terminal. Rluc could catalyze its fluorescent substrate coelenterazine (CTZ) molecules and results in emission of energy in the form of blue light with maximum wavelength at $470 \mathrm{~nm} \sim 480 \mathrm{~nm}$. The recombinant protein was further expressed and purified from bacteria Escherichia coli BL21. Afterwards, quantum dots used as the acceptor can be labeled on the N-terminal of the recombinant protein. Fig. 1 shows the BRET sensor made of quantum dots used as an acceptor in the BRET sensor and glucose sensitive protein GBP conjugated RLuc used as a donor in the sensor. 

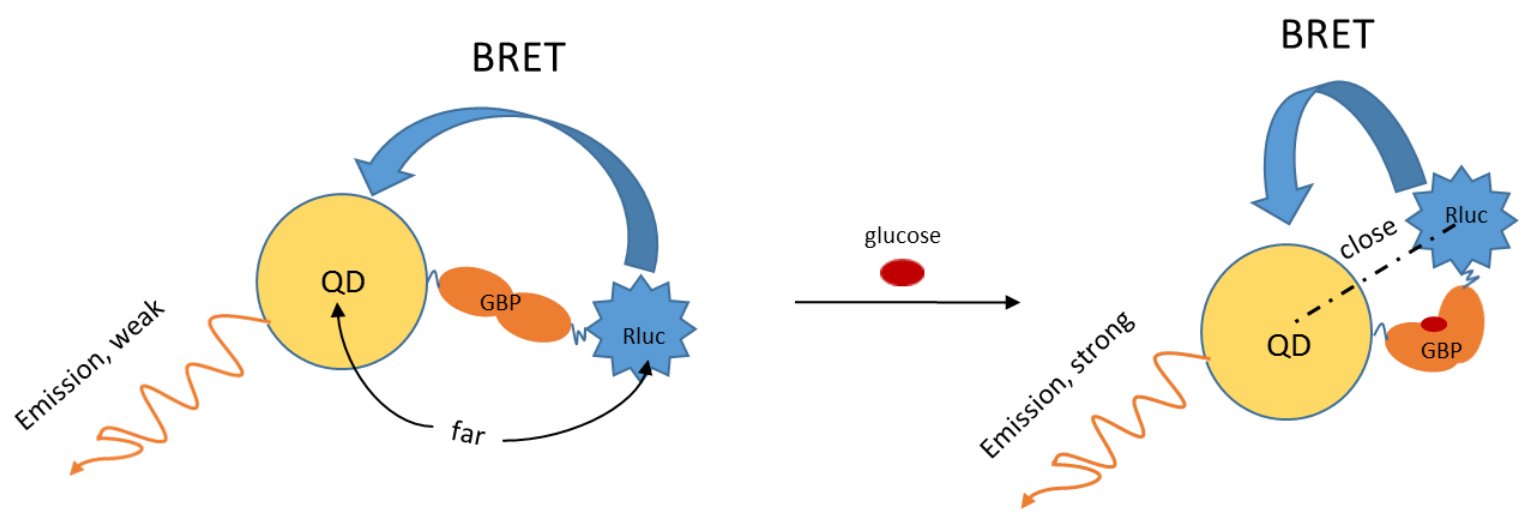

Fig. 1: The scheme of the BRET biosensor. QDs were used as the BRET acceptor and Rluc was the donor. GBP was at the middle site and will fold in the presence of glucose molecules.

\section{Experimental Material}

Unless otherwise stated, the chemicals was purchased from Sigma-Aldrich and only deionized water $(\rho=18.2 \mathrm{M} \Omega)$ was used.

\subsection{Plasmid Constructions}

Bacterial glucose binding protein (GBP) was cloned from E. coli k-12. Rluc gene was cloned from the plasmid pRLnull (Promega, Inc). A six amino acid linker was inserted to link the two proteins. Four primers were designed for construct the GBP-Rluc recombinant protein. For GBP, two primers were designed for Cloning (forward 5' TATACATATGAATAAGAAGGTGTTAACCCTGTCTGC $\quad 3{ }^{\prime} ; \quad$ reverse 5' GCTGGATCCTTTCTTGCTGAATTCAGCCAGGTTG 3'). The forward and reversed primers were introduced restriction site Nde I and BamH I (restrict enzyme) respectively (underline). In order to separate the sequence of GBP from that of Rluc, a six amino acid linker SGGGGS (bold underline) was inserted after BamH I site (underline) when designing the forward primer to amplify AAAGGATCCAGCGGTGGTGGTGGTAGCATGACTTCGAAAGTTTATGATCCAG 3'). The reverse primer for Rluc (5'TGTGCTCGAGTTGTTCATTTTT GAGAACTCGCTC 3') introduced restriction site Xho I (underline). The GBP is thus located at the upstream (N-terminal) of the fusion protein. The above PCR products were further digested with relating restriction enzyme. The plasmid pET32a (Novagen, Inc) was used to clone and express the recombinant gene. The digested DNA insert were ligated into the relating MCS (multiple cloning) site at pET32a. A six histidine tail was introduced into the GBP-Rluc recombinant protein. The pET 32a-GBP-Rluc was transformed into E. coli BL21 cells. The DNA sequence of the recombinant plasmid was confirmed by DNA sequencing (Robarts Institute, Western University).

\subsection{Protein Expression and Purification}

The bacterial cells with pET32 a-GBP-Rluc recombinant plasmid were grown overnight at $37^{\circ} \mathrm{C}$. in $5 \mathrm{~mL}$ of Luria Bertani (LB) broth containing $100 \mu \mathrm{g} / \mathrm{mL}$ ampicillin. This culture was used to further inoculate $600 \mathrm{~mL}$ of broth containing $100 \mu \mathrm{g} / \mathrm{ml}$ ampicillin, and this was grown at $37^{\circ} \mathrm{C}$. When the culture reached an OD600 of 0.375 , Isopropyl $\beta$-D-1thiogalactopyranoside (IPTG) was added to $1 \mathrm{mM}$ final concentration to induce the expression of GBP-Rluc and the bacteria were left to grow for $4 \mathrm{hrs}$ at room temperature. The cells were harvested by centrifugation at 9,000 rpm for $5 \mathrm{~min}$ at $4^{\circ} \mathrm{C}$. The pellet was resuspended in a binding solution (BS) of $20 \mathrm{mM}$ Tris/ $\mathrm{HCl}, \mathrm{pH} 7.4,500 \mathrm{mM} \mathrm{NaCl}$ and $5 \mathrm{mM}$ imidazole and sonicated on ice using 15-s bursts followed by 30-s rest for 30 cycles using a Mandel Scientific Q500 sonicator (Guelph, Canada). The suspension was centrifuged at $8,000 \mathrm{rpm}$ at $4^{\circ} \mathrm{C}$ for $30 \mathrm{~min}$ to collect the supernatant from bacterial cell pellet. The protein was purified via His-trap HP columns (GE lifescience, Inc.) by a syring pump. The column was first equilibrated with BS. The supernatant containing the protein was loaded on the column, and the column was washed with 10 column volumes of the BS. The protein was eluted using BS with a gradient of imidazole from $20 \mathrm{mM}$ to $150 \mathrm{mM}$ ) over 10 column volumes. Around 30 milliliters fractions were collected. SDS-PAGE (10\% SDS-PAGE under reducing conditions and stained with Coomassie Blue) was used to verify the fractions containing the fusion protein. Excess imidazole was removed from the combined fractions by buffer exchange with excess amount of $10 \mathrm{mM}$ PBS, pH 
7.4 using an Amicon Ultra centrifugal filter (ultra-15, MWCO $10 \mathrm{kDa}$, Millipore Inc). The resultant GBP-Rluc protein solution was stored at $-20^{\circ} \mathrm{C}$. The concentration of the protein was determined by Bicinchonici acid (BCA) protein assay (Thermo scientific Inc.).

\subsection{Synthesis of Thioglycolic Acid (TGA) Coated CdTe Quantum Dots.}

The colloidal CdTe QD solution was prepared based on the method described elsewhere.[26] Briefly, $0.4 \mathrm{mmol}$ $\mathrm{Cd}\left(\mathrm{CH}_{3} \mathrm{COO}\right) 2 \cdot 2 \mathrm{H}_{2} \mathrm{O}$ was dissolved into $60 \mathrm{ml}$ deionized water in a three-necked flask. Then $36 \mu \mathrm{lGA}$ was added into the solution. After stirring for $5 \mathrm{~min}, 0.08 \mathrm{mmol} \mathrm{Na}_{2} \mathrm{TeO}_{3}$ which was dissolved in $50 \mathrm{ml}$ deionized water was added into the above solution. After $160 \mathrm{mg}$ of $\mathrm{NaBH}_{4}$ was added into the precursor solution, the pH was adjusted with $1 \mathrm{M} \mathrm{NaOH}$ solution to 11 . Then the flask was attached to a condenser and refluxed at $100{ }^{\circ} \mathrm{C}$ under open-air conditions. The QDs with desired PL emission spectra can be obtained through controlling the reaction time. The QDs nanoparticles were washed twice by isopropanol and finally dissolved in deionized water.

\subsection{Bioconjugation of TGA Coated CdTe to GBP-Rluc}

The bioconjugation was mediated by 1-ethyl-3-(3-dimethylami-nopropyl) carbodiimide (EDC). In a typical experiment, TGA Stabilized CdTe $(10 \mu \mathrm{l}$ at $2 \mu \mathrm{mol} / \mathrm{L})$ and EDC $(10 \mu \mathrm{L}$ at $20 \mathrm{mmol} / \mathrm{L})$ were mixed in $80 \mu 1$ PBS. After the mixture were gently shaking for $10 \mathrm{~min}$ at room temperature, $50 \mu \mathrm{l}$ of $0.1 \mathrm{mmol} / \mathrm{L}$ protein was added into the above solution and incubated for another $10 \mathrm{~min}$. Afterwards, $2 \mu \mathrm{l}$ of ethanolamine was added to stop the reaction. The particles were further purified by amicon ultra-0.5 filter (100kDa, MWCO, EMD MilliporeInc.) to remove free GBP-Rluc and other small molecules.

\subsection{Characterization}

The TGA-coated CdTe quantum dots were verified by using Fourier transform infrared (FTIR) spectrophotometer (BrukerFTIR-IFS55, Germany). Bioluminescent emission spectra were collected by a Quanta Master ${ }^{\mathrm{TM}} 40$ Spectrofluorometer (HORIBA-PTI Inc., London, ON) with the step size at $0.1 \mathrm{~s}$. The bioluminescence recovery ratio (BioL ratio) was defined by following equation,

$$
\text { BRET Ratio I'= } \mathrm{I}_{\mathrm{A}} / \mathrm{I}_{\mathrm{D}}
$$

where $I_{A}$ and $I_{D}$ are the intensities of two fluorescence peaks at $565 \pm 5 \mathrm{~nm}$ and $475 \pm 5 \mathrm{~nm}$, generated from QDs and the GBP-Rluc, respectively, as the function of the concentration of aqueous glucose from $0.01-1 \mathrm{mmol} / \mathrm{L}$ in $3 \mathrm{ul}$ were measured.

\subsection{Glucose Assay}

The above GBP-Rluc-QDs conjugate solution was diluted to $400 \mu$ by PBS and then treated with $3 \mu 1$ of aqueous glucose with different concentrations from $0 \mathrm{mmol} / \mathrm{L}$ to $1 \mathrm{mmol} / \mathrm{L}$. The mixture was gently shaken for $2 \mathrm{~min}$ at room temperature. The fluorescence emissions were then measured from $410 \mathrm{~nm}$ to $640 \mathrm{~nm}$ by the fluorometer immediately following addition of $5 \mu \mathrm{L}$ of native $\mathrm{CTZ}(1 \mathrm{mg} / \mathrm{mL}$, dissolved in ethanol) to the above assay solutions. Each concentration of glucose was tested for three times.

\subsection{Animal Tear Test}

The blood glucose level of three male Sprague-Dawley rats (Charles River Laboratories, St. Constant, QC, Canada) were tested before collecting the tear. Tear fluid was collected from the ocular surface with a $1 \mu \mathrm{L}$ glass capillary tube (P1424 SIGMA). $1 \mu \mathrm{L}$ of rat tear sample were collected from each male Sprague-Dawley rats from the ocular surface with a $1 \mu \mathrm{L}$ glass capillary tube (P1424 SIGMA). The tear samples were diluted by PBS pH 7.4 to $10 \mu \mathrm{L}$. To keep the same amount and the procedure of the BRET sensor measurement, $3 \mu \mathrm{L}$ of the diluted sample was used to measure the glucose level by BRET sensor with 3 independent tests. Ethics approval was obtained through the University of Western Ontario Research Ethics Board, in accordance with Canadian Council on Animal Care guidelines. 


\section{Results and Discussion}

\subsection{Photoluminescence and FTIR Spectra of the TGA-Stabilized CdTe.}

Figure 2a shows the photoluminescence spectra of the TGA-stabilized CdTe aqueous solution taken from the refluxing reaction mixture at different intervals of time. With the prolonging of reflux time, the photoluminescence emission spectra of the CdTe NCs shifted to longer wavelengths with increasing size of the CdTe NCs as a consequence of the quantum confinement.[26] The 2 hours $(\mathrm{PL} \approx 565 \mathrm{~nm})$ QDs were chosen for the following experiments. Fig.2b shows the FTIR spectra of the TGA-stabilized CdTe. Asymmetric and symmetric stretching bands of -COO- located at 1556 and $1378 \mathrm{~cm}^{-1}$, respectively. The C-O stretch vibrational mode at $1225 \mathrm{~cm}^{-1}$ is also observed in the spectrum of TGA-QD.[27]

a

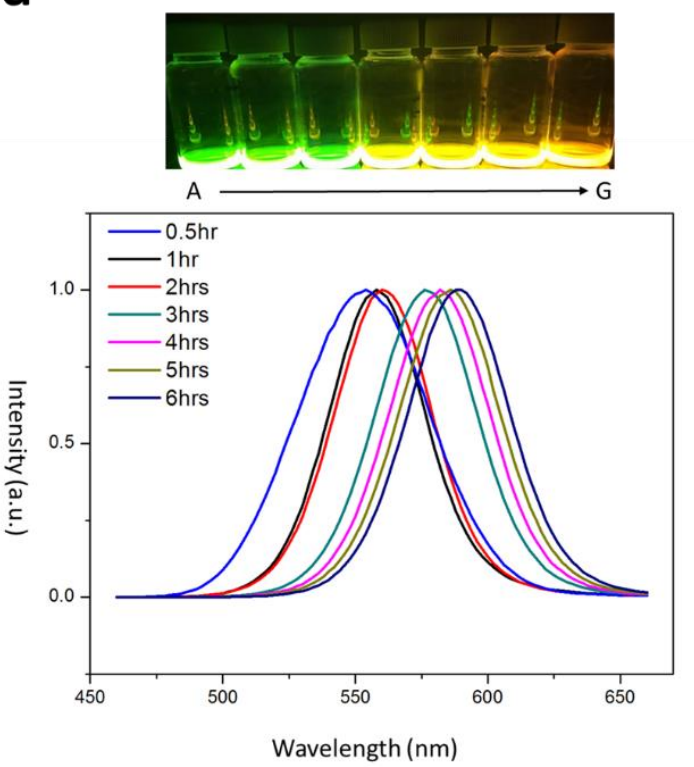

b

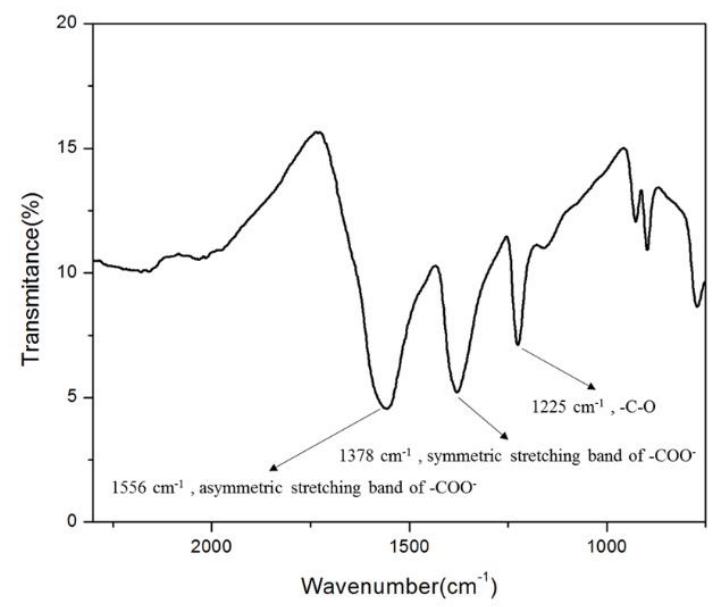

Fig. 2: (a) The image of the TGA-coated CdTe QDs under an ultraviolet lamp (top) and the photoluminescence spectra (bottom). The photoluminescence was at (A) 0.5hr, (B) 1h, (C) 2h, (D) $3 \mathrm{~h}$, (E) $4 \mathrm{~h}$, (F) 5h, (G) 6h. (b) Fourier Transform Infrared (FTIR) spectra of TGA-coated QDs.

\subsection{Protein Expression and Purification}

Currently, the most frequently used luciferases in bioimaging are from the firefly, Photinus pyralis (Fluc), and the sea pansy, Renilla reniformis (Rluc). Compared to Fluc, which is ATP dependent, Rluc ATP-independent and is only $36 \mathrm{kDa}$. Rluc uses coelenterazine as the substrate, and emits at $480 \mathrm{~nm}$. In addition, its smaller size compared with Fluc (62kda) makes it more appropriate for application in bioluminescent system.[28] Currently, the mutation of native Rluc, Rluc2 or Rluc8, have been used as the luciferase donor to improve luminescence intensity and stability. [29] However, As the luciferase donor, the sensitivity of the native Rluc was found to be better than that of Rluc2 or Rluc8.[17] Therefore, native Rluc was chosen for the construction of the BRET sensor.

The theoretical molecular sizes of Rluc and GBP were $36 \mathrm{kDa}$ and $33 \mathrm{kDa}$, respectively. Taking the $6 \times$ His-tag, and linker into consideration, the molecular size of the fusion protein was about $71 \mathrm{kDa}$. After purification, SDS-PAGE was carried out using the method of Laemmli on an acrylamide gel with $7.5 \%$ resolving gel and $4 \%$ stacking gel to identify the protein expression and purification. As indicated in Fig.2, The recombinant protein formed a band of approximately 71 $\mathrm{kDa}$ as expected. 


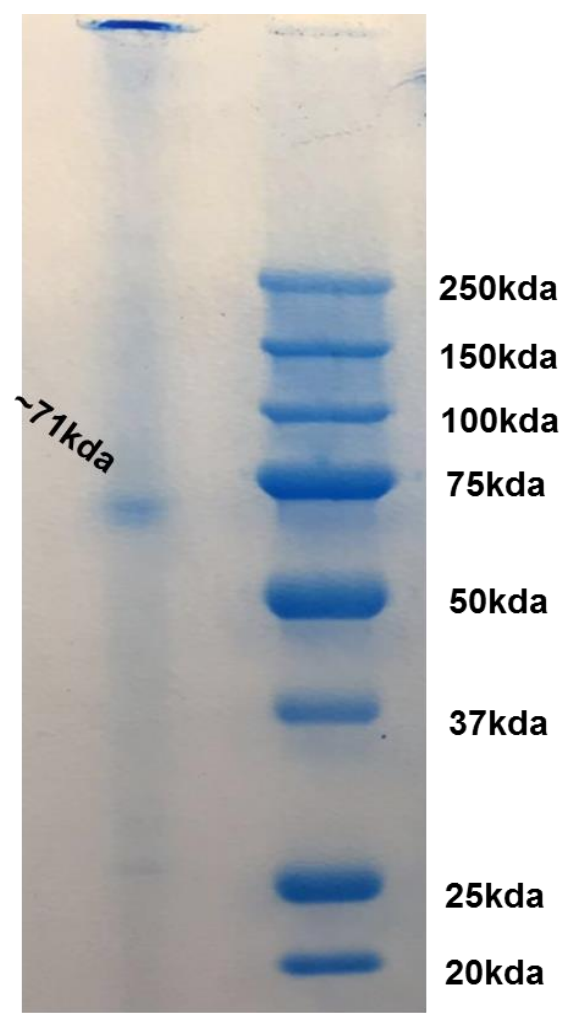

Fig. 3: SDS-PAGE characterization of recombinant protein GBP-Rluc.

\subsection{Assay Optimization}

In order to find the best performance of GBP-Rluc, different concentration of the protein, substrate and different buffer were tested. Fig. $4 \mathrm{a}$ shows the bioluminescence of $0.1 \mathrm{mg}$ protein dissolved in $400 \mu \mathrm{L}$ PBS with different amount of CTZ $(1 \mathrm{mg} / \mathrm{ml})$. The intensity increased when CTZ increased from 3 to $5 \mu \mathrm{L}$, while dropped when CTZ kept increasing to $10 \mu \mathrm{L}$. Fig. $3 \mathrm{~b}$ shows the affection of the protein amount to the bioluminescence performance. The intensity were found to drop when protein mass increased over $0.2 \mathrm{mg}$. The behaviour in different buffer: $10 \mathrm{mM}$ Tris/ $\mathrm{HCl}, \mathrm{pH} 7.4,10 \mathrm{mM}$ PBS, $\mathrm{pH} 7.4$ and $10 \mathrm{mM}$ Borate, $\mathrm{pH} 8.0$ were tested and the result was shown in Fig. 4c, which indicates the 10mM PBS, pH 7.4 is best for the assay. The decay rate of the bioluminescence was measured with a time interval at $2 \mathrm{~s}$, with different amount of protein, as shown in Fig.4d. 

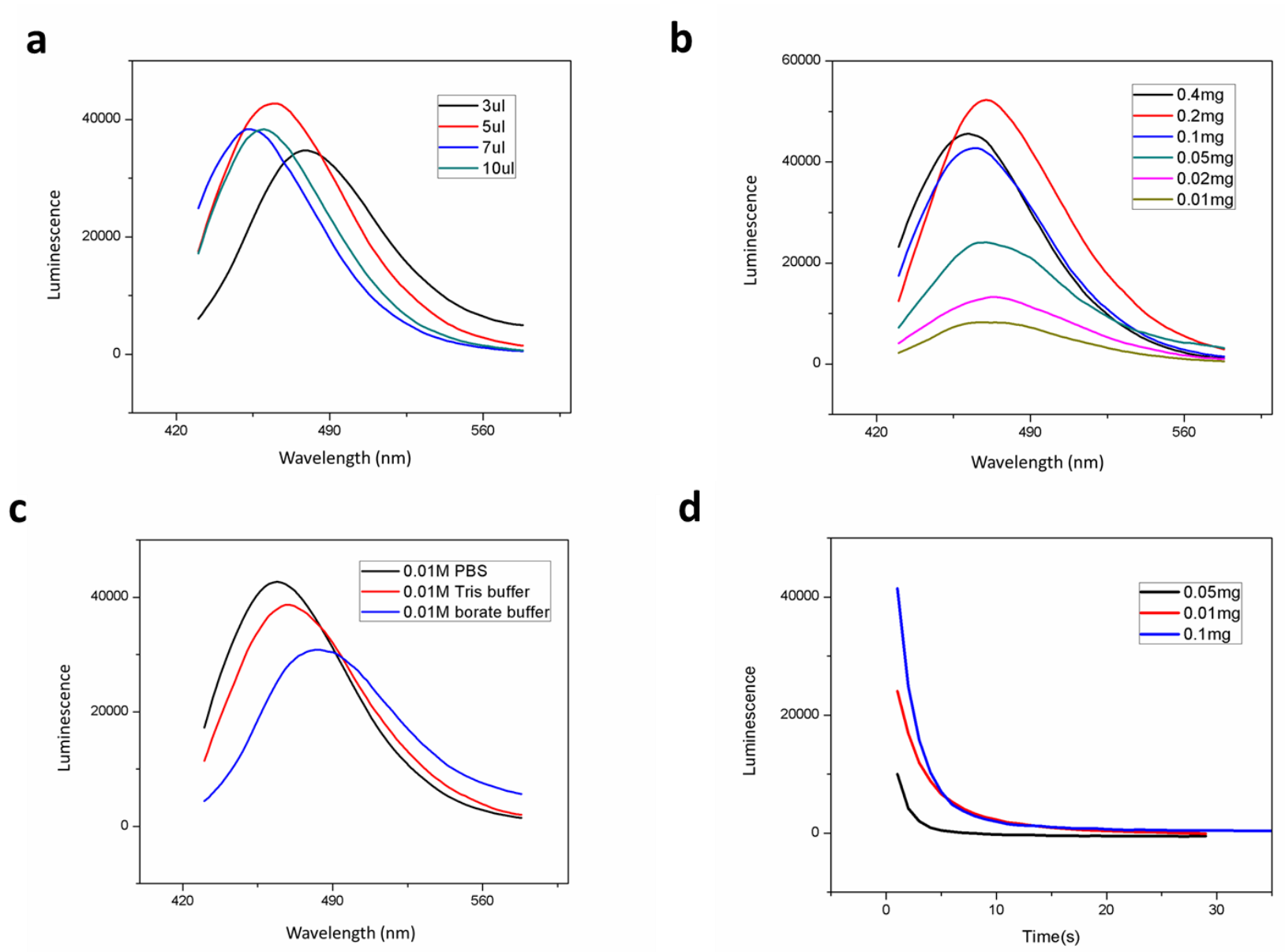

Fig. 4: The performance of GBP-Rluc in different conditions. (a) The effects of CTZ amount. (b) The effects of protein amount. (c) The effect of buffers. (d) The effect of time.

To optimal the sensor for glucose detection, Firstly the amount of EDC is fixed to be $20 \mathrm{mM}$. The ratio between the protein and quantum dots were tested at 50:1, 150:1, 250:1 and 400:1, respectively and then treated with $3 \mu \mathrm{L} 0.1 \mathrm{mmol} / \mathrm{L}$ of aqueous glucose. The intensity change $\mathrm{I}_{\mathrm{c}}$ is defined as follow:

$$
\mathrm{I}_{\mathrm{c}}=\mathrm{I}_{\mathrm{w}}-\mathrm{I}_{\mathrm{g}} / \mathrm{I}_{\mathrm{g}}
$$

Where $I_{g}$ and $I_{w}$ are the intensity ratio of the acceptor and donor before and after treated with glucose solution. As EDC mediated all the amine groups on the surface of GBP-Rluc with the carboxylic groups on the surface of CdTe, the bioconjugation process is non-site specific [23,30], the excess amount of either GBP-Rluc or QDs may affect the sensitivity of this sensor. Fig.5a shows the best sensitivity presents around the ratio at 250:1. Also, the molar ratio of EDC to QDs was also tested at 1000:1, 2500:1 5000:1, 10000:1 and 20000:1. The ratio between GBP-Rluc and QDs is mixed at 250:1 and $3 \mu \mathrm{L}$ of $0.1 \mathrm{mmol} / \mathrm{L}$ aqueous glucose was added for the test. Though the amount of EDC is key to mediate the reaction, high concentration of EDC could cause the self-crosslink of proteins and irreversible precipitation of the products which result in low signal response, as shown in Fig.5b. The best ratio between the EDC and the QDs is found to be at 10000:1. 
a

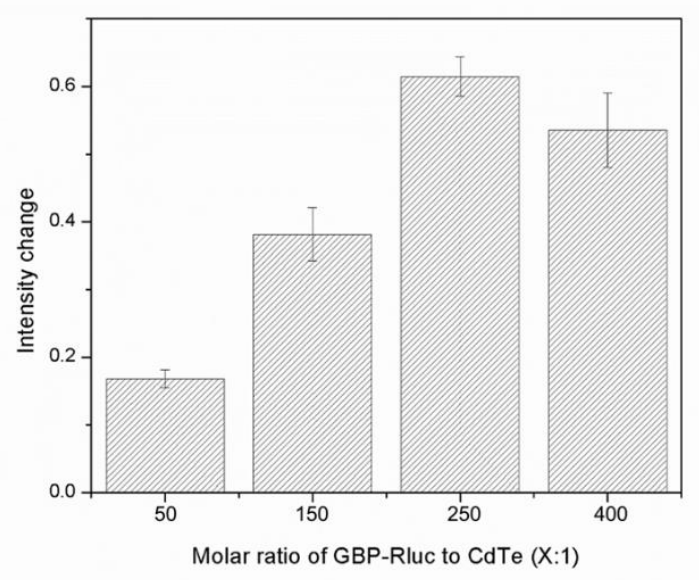

b

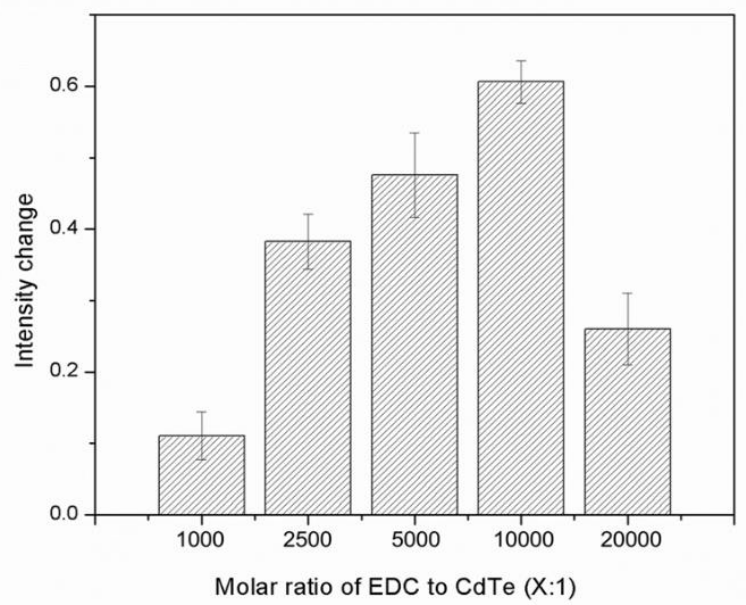

Fig. 5: Performance of designed sensor with $0.1 \mathrm{mM}$ glucose. (a) Intensity change with different ratio of EDC to QDs. (b) Intensity change with different ratio of GBP-Rluc to QDs.

\subsection{Glucose assay and Tear Test}

The capability of the nanostructured BRET sensor to measure the concentration of glucose is evaluated under the above optimized condition. Fig. 6a shows the BRET spectra with different concentration of glucose. The increase of glucose concentration results in an increasing of the BL emission intensity.

a

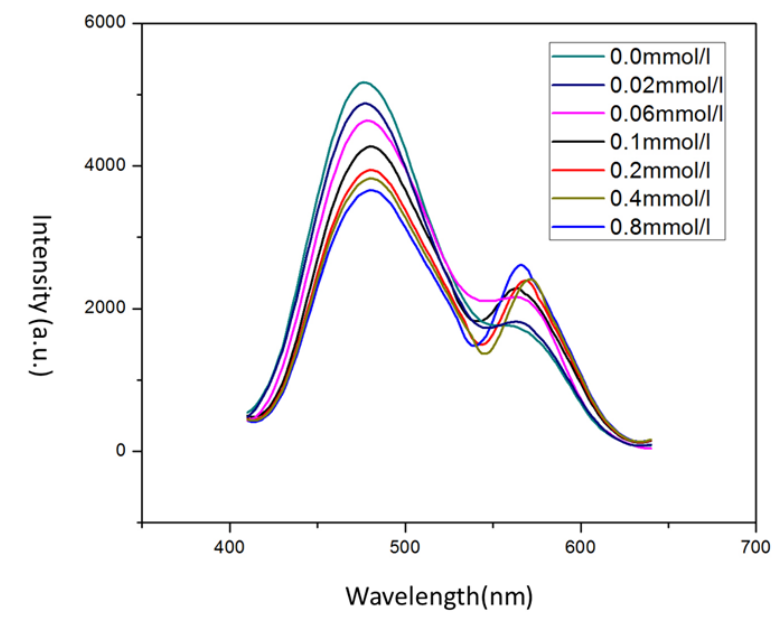

b

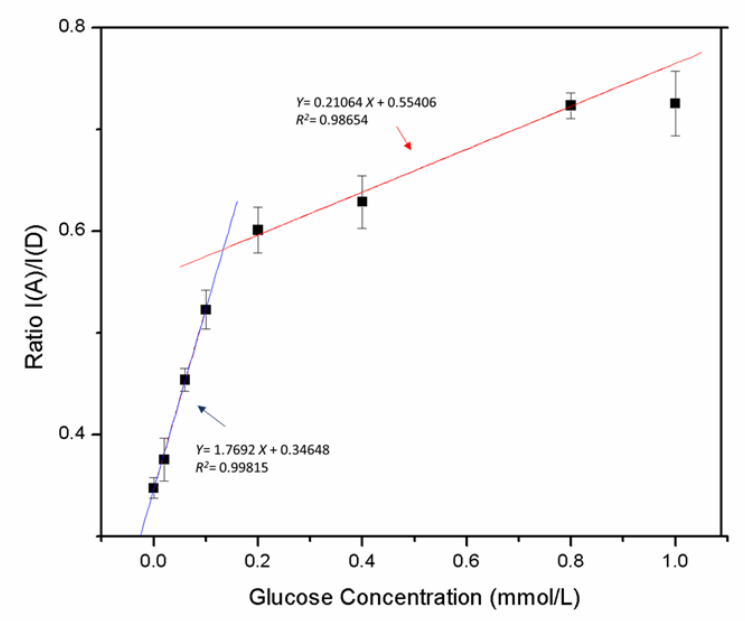

Fig. 6: (a) The BRET spectrum with different concentrations of glucose. (b) Linear relationship between BRET intensity ratio of the designed sensor and the concentration of glucose.

According to Eq.1, The BL intensity were calculated and the linear relationships between glucose concentration and the BL intensity are found in the ranges of $0-0.1 \mathrm{mM}$ and $0.2 \mathrm{mM}-0.8 \mathrm{mM}$, respectively as shown in Fig. $6 \mathrm{~b}$. The limit of detection (LOD) for this assay is secured to $0.03 \mathrm{mM}$ based on $3 \sigma$ from three independent measurements. The relationship between the concentration of glucose and the intensity ratio in the two ranges mentioned above can be expressed as the following equations, respectively. 


$$
\begin{gathered}
\boldsymbol{Y}=1.7692 \boldsymbol{X}+0.34648 \\
\boldsymbol{Y}=0.21064 \boldsymbol{X}+0.55406
\end{gathered}
$$

\section{Conclusion}

In summary, we developed a novel BRET biosensor for monitoring the tear glucose. CdTe was used as acceptor and luciferase Rluc was used as the bioluminescence donor. The distance between the donor and the acceptor is mediated by the folding of GBP, which is at the upstream of Rluc, with existence of glucose molecules. The ratio of the acceptor (QDs) and the donor (Rluc) has been observed to increase with the increasing of the glucose concentration and a similar linear relationship is found both in the range from $0 \mathrm{M}$ to $0.1 \mathrm{mM}$ and from $0.2 \mathrm{mM}$ to $0.8 \mathrm{mM}$. The limit of detection is as low as $0.03 \mathrm{mmol} / \mathrm{L}$.

\section{Acknowledgements}

Authors are thankful for the financial support from the Grand Challenges Canada- Rising Star, Canada Innovation Fund-Leaders Opportunity Fund and Natural Sciences and Engineering Research Council of Canada (NSERC).

\section{References}

[1] C. Chen, X. L. Zhao, Z. H. Li, Z. G. Zhu, S. H. Qian, and A. J. Flewitt, "Current and Emerging Technology for Continuous Glucose Monitoring," Sensors (Basel), vol. 17, no. 1, 2017.

[2] X. Huang, L. Xie, X. Lin, and B. Su, "Detection of Metoprolol in Human Biofluids and Pharmaceuticals via IonTransfer Voltammetry at the Nanoscopic Liquid/Liquid Interface Array," Anal Chem, vol. 89, no. 1, pp. 945-951, 2017.

[3] X. Gu, H. Wang, Z. D. Schultz, and J. P. Camden, "Sensing Glucose in Urine and Serum and Hydrogen Peroxide in Living Cells by Use of a Novel Boronate Nanoprobe Based on Surface-Enhanced Raman Spectroscopy," Anal Chem, vol. 88, no. 14, pp. 7191-7, 2016.

[4] L. Chen, Y. Bao, J. Denstedt, and J. Zhang, "Nanostructured bioluminescent sensor for rapidly detecting thrombin," Biosens Bioelectron, vol. 77, pp. 83-9, 2016.

[5] C. S. Hyunjae Lee, Yong Seok Hong, Min Sung Kim, Hye Rim Cho,Taegyu Kang, Kwangsoo Shin, Seung Hong Choi,Taeghwan Hyeon, Dae-Hyeong Kim, "Wearable/disposable sweat-based glucose monitoring device with multistage transdermal drug delivery module," SCIENCE ADVANCES, vol. 3, no. 3, pp. e1601314, 2017.

[6] R. Guy, "Diagnostic devices: Managing diabetes through the skin," Nat Nanotechnol, vol. 11, no. 6, pp. 493-4, 2016.

[7] H. Lee, T. K. Choi, Y. B. Lee, H. R. Cho, R. Ghaffari, L. Wang, H. J. Choi, T. D. Chung, N. Lu, T. Hyeon, S. H. Choi, and D. H. Kim, "A graphene-based electrochemical device with thermoresponsive microneedles for diabetes monitoring and therapy," Nat Nanotechnol, vol. 11, no. 6, pp. 566-72, 2016.

[8] D. Holmes, "Innovation: Sweat-sensing patch for glucose monitoring and drug delivery," Nat Rev Endocrinol, vol. 12, no. 5, pp. 247, 2016.

[9] E. Gabriel, P. Garcia, F. Lopes, and W. Coltro, "Paper-Based Colorimetric Biosensor for Tear Glucose Measurements," Micromachines, vol. 8, no. 4, pp. 104, 2017.

[10] T. Arakawa, Y. Kuroki, H. Nitta, P. Chouhan, K. Toma, S. Sawada, S. Takeuchi, T. Sekita, K. Akiyoshi, S. Minakuchi, and K. Mitsubayashi, "Mouthguard biosensor with telemetry system for monitoring of saliva glucose: A novel cavitas sensor," Biosens Bioelectron, vol. 84, pp. 106-11, 2016.

[11] F. J. A. a. V. Huerva, "Noninvasive Continuous Monitoring of Tear Glucose Using Glucose-Sensing Contact Lenses," OPTOMETRY AND VISION SCIENCE, vol. 93, no. 4, pp. 426-434, 2015.

[12] L. Chen, W. H. Tse, Y. Chen, M. W. McDonald, J. Melling, and J. Zhang, "Nanostructured biosensor for detecting glucose in tear by applying fluorescence resonance energy transfer quenching mechanism," Biosens Bioelectron, vol. 91, pp. 393-399, 2017.

[13] R. Chen, Z. Jin, and L. A. Colon., "Analysis of tear fluid by CE/LIF: a noninvasive approach for glucose monitoring," Journal of capillary electrophoresis vol. 3, no. 5, pp. 243-248, 1995. 
[14] J. T. Baca, D. N. Finegold, and S. A. Asher, "Tear Glucose Analysis for the Noninvasive Detection and Monitoring of Diabetes Mellitus," The Ocular Surface, vol. 5, no. 4, pp. 280-293, 2007.

[15] D. K. S. A. G. S. SARIN, "Tear glucose levels in normal people and in diabetic patients," British Journal of Ophthalmology,, vol. 64, pp. 693-695, 1980.

[16] X. Yu, K. Wen, Z. Wang, X. Zhang, C. Li, S. Zhang, and J. Shen, "General Bioluminescence Resonance Energy Transfer Homogeneous Immunoassay for Small Molecules Based on Quantum Dots," Anal Chem, vol. 88, no. 7, pp. 3512-20, 2016.

[17] H. Dacres, M. Michie, J. Wang, K. D. Pfleger, and S. C. Trowell, "Effect of enhanced Renilla luciferase and fluorescent protein variants on the Forster distance of Bioluminescence resonance energy transfer (BRET)," Biochem Biophys Res Commun, vol. 425, no. 3, pp. 625-9, 2012.

[18] H. Dacres, J. Wang, V. Leitch, I. Horne, A. R. Anderson, and S. C. Trowell, "Greatly enhanced detection of a volatile ligand at femtomolar levels using bioluminescence resonance energy transfer (BRET)," Biosens Bioelectron, vol. 29, no. 1, pp. 119-24, 2011.

[19] M. Kumar, D. Zhang, D. Broyles, and S. K. Deo, "A rapid, sensitive, and selective bioluminescence resonance energy transfer (BRET)-based nucleic acid sensing system," Biosens Bioelectron, vol. 30, no. 1, pp. 133-9, 2011.

[20] N. K. V. Vyas, M. N. Quiocho, F. A., "Sugar and signal-transducer binding sites of the Escherichia coli galactose chemoreceptor protein," Science, vol. 242, pp. 1290-1295, 1988.

[21] J. A. Gerald L. Hazelbauer, "Role of the Galactose binding protein in chemotaxis of Escherichia coli toward galactose," Nature New Biology, vol. 230, 1971.

[22] L. T. Xudong Ge, and Govind Rao, "Dual-Labeled Glucose Binding Protein for Ratiometric Measurements of Glucose," Anal. Chem., vol. 76, pp. 1403-1410, 2004.

[23] H. V. Hsieh, Z. A. Pfeiffer, T. J. Amiss, D. B. Sherman, and J. B. Pitner, "Direct detection of glucose by surface plasmon resonance with bacterial glucose/galactose-binding protein," Biosensors and Bioelectronics, vol. 19, no. 7, pp. 653-660, 2004.

[24] C. Tiangco, D. Fon, N. Sardesai, Y. Kostov, F. Sevilla, G. Rao, and L. Tolosa, "Fiber optic biosensor for transdermal glucose based on the glucose binding protein," Sensors and Actuators B: Chemical, vol. 242, pp. 569576, 2017.

[25] M. M. El-Sayed, S. R. Brown, K. Mupparapu, and L. Tolosa, "The effect of pH on the glucose response of the glucose-galactose binding protein L255C labeled with Acrylodan," Int J Biol Macromol, vol. 86, pp. 282-7, 2016.

[26] S. Wu, J. Dou, J. Zhang, and S. Zhang, "A simple and economical one-pot method to synthesize high-quality water soluble CdTe QDs," Journal of Materials Chemistry, vol. 22, no. 29, pp. 14573, 2012.

[27] Y. K. Qiang Wang, Yuwen Wang, Gyehwa Shin, Chada Ruengruglikit, and Qingrong Huang, "Luminescent Properties of Water-Soluble Denatured Bovine Serum Albumin-Coated CdTe Quantum Dots," J. Phys. Chem. B vol. 110, no. 110, pp. 16860-16866, 2006.

[28] A. M. Loening, T. D. Fenn, A. M. Wu, and S. S. Gambhir, "Consensus guided mutagenesis of Renilla luciferase yields enhanced stability and light output," Protein Eng Des Sel, vol. 19, no. 9, pp. 391-400, 2006.

[29] M. K. So, C. Xu, A. M. Loening, S. S. Gambhir, and J. Rao, "Self-illuminating quantum dot conjugates for in vivo imaging," Nat Biotechnol, vol. 24, no. 3, pp. 339-43, 2006.

[30] A. M. Loening, T. D. Fenn, and S. S. Gambhir, "Crystal structures of the luciferase and green fluorescent protein from Renilla reniformis," J Mol Biol, vol. 374, no. 4, pp. 1017-28, 2007. 